\title{
Itinerários terapêuticos e construção de projetos terapêuticos cuidadores
}

\section{Therapeutic itinerary and the construction of car- ing therapeutic projects}

\author{
Elisabete Ferreira Mângia ${ }^{1}$, Melissa Tieko Muramoto
}

\begin{abstract}
MÂNGIA, E. F.; MURAMOTO, M. T. Itinerários terapêuticos e construção de projetos terapêuticos cuidadores. Rev. Ter. Ocup. Univ. São Paulo, v. 19, n. 3, p. 176-182, set./dez. 2008.

RESUMO: A construção de novas formas de cuidado em saúde nos coloca diante de um contexto de mudanças e desafios provocados pela consolidação do SUS, sua manutenção e aprimoramento. Nesse sentido, faz-se necessário a incorporação de novos recursos, capazes de contribuírem com a construção e formulação de propostas de intervenção que sejam centradas no indivíduo e que possam considerar sua interação com seu contexto e historia de vida. Utilizando o modelo proposto por Merhy, no qual o autor defende que as novas abordagens devam ser construídas a partir do universo relacional, onde é possível produzir o "cuidado que cuida", pautado particularmente nas tecnologias leves, apresentamos o conceito de itinerários terapêuticos e discutimos a compreensão deste fenômeno como um recurso que possibilita a construção de projetos terapêuticos cuidadores, que levem em consideração as experiências, significados, trajetórias, desejos e necessidades da pessoa na elaboração de seu próprio processo de tratamento.
\end{abstract}

DESCRITORES: Assistência à saúde. Acesso aos serviços de saúde. Avaliação de programas e projetos de saúde.

1. Profa. Dra. do Curso de Terapia Ocupacional Departamento de Fisioterapia, Fonoaudiologia e Terapia Ocupacional da Faculdade de Medicina da Universidade de São Paulo - FMUSP.

2. Mestre em Ciências da Reabilitação pela Faculdade de Medicina da Universidade de São Paulo. Terapeuta Ocupacional do Departamento de Fisioterapia, Fonoaudiologia e Terapia Ocupacional da FMUSP.

Endereço para correspondência: Departamento de Fisioterapia, Fonoaudiologia e Terapia Ocupacional da FMUSP. Rua Cipotânea, 51. Cidade Universitária. São Paulo, SP. CEP: 05360-160. 


\section{INTRODUÇÃO}

$\mathrm{A}$ construção de um novo modelo assistencial se coloca como prioridade no contexto das mudanças e desafios colocados e provocados pelo processo de consolidação do SUS, sua manutenção e aprimoramento. Para o campo da saúde mental esse desafio é particularmente relevante e dele depende a possibilidade de constituição de uma rede de serviços substitutivos e comprometidos com as diretrizes da integralidade, da universalidade, do acesso e particularmente, com a cidadania dos usuários.

A nova lógica está ancorada na crítica ao modelo biomédico, seus limites e conseqüente necessidade de superação desse paradigma. O modelo que se critica é a matriz constitutiva de todo o campo da saúde e suas múltiplas áreas, competências e núcleos de formação profissional.

Saraceno considera o modelo biomédico como tributário de um paradigma positivista, linear, individualista e a-histórico que trabalha com um princípio determinista básico - para cada doença existe uma causa e se propõe um tratamento -. Tal princípio é extremamente simplificador na forma como compreende o sofrimento e formula respostas. Propõe intervenções centradas no indivíduo e particularizadas na doença e desconsidera a interação entre indivíduo e seu contexto e história. Essa crítica vem sendo assumida e desenvolvida por muitos autores e no Brasil já se constitui como importante núcleo de investigação e projetos da saúde coletiva e de diversos núcleos profissionais (SARACENO, 1998).

Nesse cenário destacam-se as contribuições de Merhy, que compreende que um dos nós críticos a serem superados na atualidade é o das práticas assistenciais centradas nos procedimentos profissionais, como marca da ação ainda hegemônica do paradigma biomédico.

Discutindo o conceito de integralidade alerta que somos nós que podemos dotar esse conceito de sentido a depender das práticas de saúde que protagonizamos. Como fazer isso em nosso contexto no qual o cuidado em saúde ainda se estrutura a partir dos territórios de cada profissão e de acordo com o modelo biomédico, ainda hegemônico, que tem como uma de suas características centrais a oferta de práticas centradas em procedimentos profissionais?

Para Merhy, o único caminho possível é o de permitir que o nosso agir profissional seja reconfigurado, desterritorializado e esse processo só pode ocorrer como ato coletivo no contexto do trabalho em equipe. O profissional deveria refletir sobre a oferta que faz se colocando no lugar de quem recebe ou receberia o cuidado e criticar a oferta focada em parcialidades, que fragmenta o sujeito em diversos procedimentos técnicos. Essa fragmentação não permite reconhecer o usuário como um sujeito de desejos, possibilidades, necessidades, direitos e capacidade de apropriação e invenção de seu projeto de saúde e, mais do que isso, não o reconhece como parceiro na construção de projetos terapêuticos (MERHY, 2005).

Para Merhy, o desenvolvimento de projetos terapêuticos de novo tipo requer a superação desse modo de cuidar - centrado no procedimento - e que no caso da saúde mental tem características bem peculiares: pode ser identificado não apenas nas práticas clínicas tradicionais, médicas ou psicoterápicas, mas também no trabalho centrado em oficinas; em atividades culturais e de lazer descontextualizadas da vida e das necessidades dos usuários, ou nas práticas artísticas orientadas apenas pelo desejo, gosto ou formação especialística dos técnicos. Para tanto, acredita ser necessário explorar as "porosidades localizadas de modo mais explícito nas relações operadas pelas tecnologias leves, para dar conta dos encontros e relações com o outro: o usuário" (MERHY, 2005).

Esse território relacional não se localiza em nenhum campo tecnológico específico, sendo um espaço ocupado por todos e configurando ao mesmo tempo o que ele chama de "dimensão cuidadora" das práticas de saúde. Seriam nessas porosidades que as profissões poderiam se encontrar desterritorializadas e nelas encontrar o usuário (MERHY, 2005).

Para o autor a "dimensão cuidadora" se estabelece a partir do cotidiano de todos os profissionais e serviços no contexto dos encontros com os usuários. A qualidade desses encontros, suas características relacionais e processos que são por eles desencadeados definem um campo de ação ao mesmo tempo técnico e ético que poderá ou não contribuir para o processo de defesa da vida e construção de um novo paradigma de assistência (MERHY, 1997).

Com indicação das possibilidades e pedidos inscritos nessa dimensão, o autor nos oferece alguns exemplos que facilitam a compreensão daquilo que está em causa nessa perspectiva:

\footnotetext{
"Relação intercessora com o mundo subjetivo do usuário e o seu modo de representar e construir necessidades de saúde; Relação acolhedora que inclua o usuário como sujeito de saberes e práticas no campo das ações de saúde;

Relação acolhedora com o usuário que permita produzir vínculos e responsabilizações entre todos que estão implicados com os atos de saúde;

Relação que permita articulação de saberes para compor as valises tecnológicas e os projetos de intervenções em saúde
} 
do modo mais amplo possível;

Relação que possibilita o encontro dos núcleos profissionais a partir do mundo do usuário como lógica capturante das outras lógicas, e não ao contrário;

Relação que se compromete com a finalidade dos atos de saúde como efetivos para a qualificação do viver individual e coletivo;

Relação que se orienta pela aposta no agenciamento de sujeitos morais implicados com a defesa da vida individual e coletiva;

Relação de inclusão cidadã, que opera na construção de autonomias e não de clones no campo da produção dos sujeitos sociais; entre outras". (Merhy, 2005).

Essa abordagem nos permite concluir que é só no universo relacional que se pode produzir o "cuidado que cuida" e este está situado, em relação ao processo de trabalho em saúde, no espaço das tecnologias leves.

É oportuno aqui definirmos o sentido desse conceito e o contexto no qual se coloca, que é o da análise do processo de trabalho em saúde, descrito por Merhy (1997) como composto pelo "Trabalho Morto" - que consiste na dimensão instrumental do trabalho, seus recursos tecnológicos e sobre a existência de um investimento pregresso de trabalho sobre algum desses recursos -, e o "Trabalho Vivo em Ato" - que consiste no trabalho no momento de sua realização; é o agir propriamente dito, marcado fortemente pela dimensão relacional e pela qualidade do encontro entre serviços, profissionais e usuários. Tais elementos constituem a Composição Técnica do Trabalho (CTT), que pode ser definida como a razão entre seus dois componentes no processo de trabalho.

A transição tecnológica esperada na atualidade é a de uma CTT configurada pelo predomínio do Trabalho Vivo em Ato e a relação e o equilíbrio entre esses dois componentes pode sugerir o quanto o processo analisado está mais distante ou mais próximo dessa expectativa.

Mais precisamente, no desempenho do cuidado em saúde, os serviços e profissionais se utilizam de três tipos de tecnologias: as tecnologias duras (equipamentos, máquinas, normas e estruturas relacionais), as tecnologias leve-duras (os saberes estruturados que operam no processo de trabalho) e, finalmente, as tecnologias leves, que são as tecnologias relacionais, responsáveis pela construção de vínculos, acolhimento e demais aspectos relacionais do trabalho, ou seja, são elas que definem e qualificam o encontro entre profissionais e usuários. São as tecnologias leves o grande espaço de construção de novas formas de cuidar, como já foi dito acima (MERHY, 1997; MERHY, FRANCO, 2003).
Para Merhy e Franco, o novo modelo assistencial, além de oferecer aos usuários os recursos tecnológicos necessários, deveria contribuir com um processo baseado nas "tecnologias leves, relacionais e a produção do cuidado de forma integralizada, operando em "linhas de cuidado" por toda extensão dos serviços de saúde, centrado nas necessidades dos usuários" (MERHY, FRANCO, 2003).

As novas redes de serviços de saúde mental, a estratégia de saúde da família, a, implantação dos Núcleos de Apoio a Saúde da Família, são exemplos de projetos que buscam construir novas maneiras de produzir saúde. Em muitas experiências se percebe o esforço na formulação e desenvolvimento do trabalho em equipe, orientado pelas necessidades dos usuários e não mais na fragmentação das ações técnicas específicas de cada corporação. Mas ainda em muitas delas se pode encontrar a reprodução de relações tradicionais entre serviços, equipes e usuários, que necessitam de avaliação e investimento, prático e formativo. Nesse sentido é interessante situarmos um aspecto que deve compor o novo desenho das práticas e que consistiria nas novas possibilidades de oferta de escuta ao usuário e de promoção real de sua participação ativa no processo de construção, desenvolvimento e avaliação das práticas de cuidado.

Neste trabalho buscamos contribuir com a discussão sobre a construção de projetos terapêuticos situando o leitor sobre a importância da investigação sobre os itinerários terapêuticos como recurso e ferramenta na construção de projetos terapêuticos cuidadores e no desenvolvimento das estratégias de pesquisa nesse campo. Pra isso se toma como ponto de partida a apresentação desse campo teórico e sua inserção e importância para o debate atual sobre as práticas de saúde.

\section{Construção de Itinerários terapêuticos, narrativas e acesso ao conhecimento sobre processo saúde doença.}

Original dos campos da sociologia e antropologia, a coleta de narrativas sobre o processo saúde doença tem sido objeto de muitos estudos contemporâneos do campo da saúde e mostram que as relações sociais ou mais precisamente, as redes sociais definem a forma como a doença é compreendida, expressada e vivida pelos sujeitos. Informa também sobre como os tratamentos propostos são avaliados, experimentados, modificados, aceitos ou abandonados, revelando a importância da mudança do olhar dos profissionais sobre a participação dos usuários no processo de produção do cuidado (ALVES; SOUZA, 1999; MÂNGIA; MURAMOTO, 2009, 2005). 
O estudo sobre itinerários terapêuticos aponta para a importância da experiência vivida pelos sujeitos no processo de enfermidade e a multiplicidade de caminhos e escolhas presentes nesse processo (ALVES; SOUZA, 1999).

A compreensão sobre como as pessoas e os grupos sociais realizam escolhas e aderem ou não aos tratamentos, ou seja, como constroem seus itinerários terapêuticos, é fundamental para orientar as novas práticas em saúde. No geral, as dimensões relativas aos contextos de vida dos usuários e suas histórias escapam aos serviços e aos profissionais de saúde, embora sejam elas que definem as possibilidades de oferta e acesso aos serviços de saúde.

O acesso a essa experiência só é possível no contexto relacional e na possibilidade dos sujeitos contarem suas histórias orais. As narrativas são a forma de acesso aos itinerários terapêuticos (ALVES; SOUZA, 1999).

Para Alves et al. (1999) a pesquisa com narrativas tem como elemento central o conceito de experiência, que diz respeito a forma como as pessoas, inseridas em determinados contextos, reconhecem, vivenciam e produzem respostas no enfrentamento das situações colocadas pela doença.

A experiência de cada sujeito é permanentemente definida e modificada pelo agir cotidiano e pela busca de soluções práticas para os processos de adoecimento e não pode ser sintetizado em um sistema coerente e ordenado de idéias. Além disso, as formas como os sujeitos explicam tais processos não mostram coerência com as ações que desenvolvem.

Tais dimensões não deveriam ser negligenciadas pelas equipes de saúde e mais do que isso, deveriam fazer parte das estratégias de cuidado, de forma a tornar o reconhecimento das experiências singulares o motor da construção de projetos de cuidado.

Assumir a diretriz da construção de projetos terapêuticos centrados nas necessidades das pessoas e contextualizados em suas histórias, necessidades e desejos, requer o desenvolvimento de novas modalidades e critério de avaliação e também de modelagens diferenciadas das práticas multiprofissionais (MÂNGIA; MURAMOTO, 2007; MÂNGIA; YASUTAKI, 2007).

A sugestão da utilização de uma nova linguagem, centrada na saúde e não mais da doença pode ser bem encaminhada quando se opta pela utilização da nova Classificação Internacional de Funcionalidade, Incapacidade e Saúde, proposta pela Organização Mundial de Saúde e não mais pela avaliação que utiliza apenas os critérios do CID-10. Ela permite uma apreensão global dos sujeitos, descentrada da doença e centrada em componentes da saúde. Ela orienta o olhar para os contextos: de vida do usuário, de relações e interações interpessoais; de relações familiares; de atividades cotidianas, de estudo e trabalho, dentre outros. Sua utilização pode ancorar as equipes tanto técnica como teoricamente para o desenvolvimento de um novo repertório avaliativo muito diferenciado daquele tradicionalmente desenvolvido no contexto formativo tradicional e fundamental para o desenvolvimento de novas abordagens (MÂNGIA et al., 2008).

O processo de produção de projetos terapêutico cuidadores requer fundamentalmente a reorientação do tipo de escuta oferecida ao usuário e a possibilidade de compreensão sobre sua história, assim deve fazer parte do processo o espaço para a expressão desses elementos. Sobre eles, os técnicos deveriam aprender primeiramente o respeito a alteridade e evitar o risco da interpretação apressada sobre o sofrimento do outro, no geral subsumida a linguagem técnica e redutiva.

Nesse sentido a postura etnográfica na escuta dos itinerários terapêuticos nos parece adequada, mais do que as posturas interpretativas oferecidas pelas disciplinas do campo psi, que tendem a colocar o técnico no alto de um suposto saber sobre a subjetividade do outro, carregado de seus próprios valores pessoais, culturais e estéticos, e que tende a reproduzir aquilo que tão bem mostrou um conhecido poeta da MPB; "narciso acha feio o que não é espelho" (MÂNGIA, MURAMOTO, 2006, 2007; MÂNGIA, 2002).

\section{Itinerários Terapêuticos: apontamentos teóricos}

No Brasil, a discussão sobre itinerários terapêuticos é recente e ainda conta com a contribuição de poucos autores. Dentre eles, o grupo composto por Paulo César B. Alves, Iara Maria A. Souza e Miriam C. M. Rabelo têm como contribuição mais marcante a organização do livro Experiência de Doença e Narrativa, que toma como principal objeto os processos sociais envolvidos na experiência e enfrentamento da doença mental. O debate e textos encontrados no livro partiram dos resultados de pesquisa realizada pelo Núcleo de Estudos em Ciências Sociais e Saúde (ECSAS), da Universidade Federal da Bahia, com os habitantes do Nordeste de Amaralina, bairro situado no município de Salvador, BA.

Para esses autores, o itinerário terapêutico pode ser compreendido como "um conjunto de planos, estratégias e projetos voltados para um objeto preconcebido: o tratamento da aflição.” (p.133). A literatura sobre o tema tem como principal objetivo compreender e analisar os 
"processos pelos quais os indivíduos ou grupos sociais escolhem, avaliam e aderem (ou não) a determinadas formas de tratamento." (ALVES, SOUZA, 199, p.125).

Assim como acontece com outros conceitos do campo das ciências sociais, é possível encontrar diversas explicações e definições para o conceito de itinerário terapêutico. Tal polissemia torna sua definição uma tarefa complexa e, segundo os autores, pode produzir uma redução do fenômeno a interpretações de caráter meramente explicativo.

Alves e Souza apontam que a análise dos itinerários terapêuticos não pode ser reduzida ao estudo sobre a disponibilidade de serviços e/ou ofertas de procedimentos de saúde. Criticam a abordagem adotada pela maior parte dos estudos sobre o tema, pois consideram que tais estudos operam com premissas previamente estabelecidas, tais como escolha racional, valores culturais, estruturas de redes sociais ou modelos explicativos para interpretar como as pessoas ou grupos constituem suas distintas trajetórias na busca de tratamentos para suas aflições. Esses elementos são insuficientes, na medida em que se mostram limitados para considerar a influência exercida pelo contexto sociocultural do individuo no momento de escolha do tratamento. O individuo só é capaz de construir sua trajetória a partir de um campo de possibilidades socioculturais, que são viabilizadas durante o percurso de busca e realização de tratamentos e são marcadas por projetos distintos, por vezes até contraditórios. Segundo os autores,

... na busca de uma generalidade, de uma suposta ordem, os estudiosos do itinerário terapêutico subsumiram os complexos processos que envolvem a escolha de um tratamento a um modelo de conhecimento cientifico que reduz as ações humanas a uma 'geometria do vivido' (ALVES; SOUZA, 1999, p.136).

Para evitar essa redução, os autores elaboraram importantes precauções teórico-metodológicas e sugerem que os estudos sobre itinerários terapêuticos devam priorizar a aproximação em relação ao conhecimento dos procedimentos utilizados pelos atores para interpretarem suas experiências e decidirem sobre suas ações. Isso porque a escolha por um tratamento não é definida apenas por um conjunto de estruturas cognitivas que possam ser consideradas como única fonte de significados utilizados nessas escolhas. Estas são o resultado de múltiplas negociações realizadas no contexto relacional dos sujeitos e dessa forma expressam sínteses parciais que emergem de um campo de possibilidades histórica e culturalmente defini- das. O sujeito dessas ações compartilha um conjunto de "crenças e receitas práticas para lidar com o mundo" e o trabalho interpretativo do pesquisador encontra dimensões subjetivas dificilmente compreensíveis, pois esse universo compartilhado não determina as ações de forma homogênea (ALVES; SOUZA, 1999, p.132-33). Assim,

... torna-se necessário que toda análise sobre itinerário terapêutico ponha em evidencia as experiências, as trajetórias e os próprios projetos individuais, formulados e elaborados dentro de um campo de possibilidades, circunscrito histórica e culturalmente, tanto em termos da própria noção de individuo como dos temas, prioridades e paradigmas culturais existentes. (ALVES; SOUZA, 1999, p.132).

As definições da situação e a natureza das relações intersubjetivas no processo de tomada de decisão são aspectos essenciais e interligados que trazem reformulações aos conceitos iniciais sobre itinerários terapêuticos.

Outro aspecto relevante é que o itinerário terapêutico não deve ser compreendido como resultado de um plano esquematizado e predeterminado. Dessa forma, a unidade articulada só pode ser evidenciada no processo no qual a pessoa, ao narrar sua experiência passada, pode interpretála e avaliá-la de acordo com o seu momento atual. Assim, o itinerário terapêutico está relacionado concomitantemente a experiência vivida e ao próprio discurso sobre essa experiência. Dessa forma, há um permanente processo de atribuição de significados que é reformulado no interior de cada situação vivida e em relação ao contexto relacional no qual ocorre. A produção discursiva sobre os itinerários terapêuticos é, pois, circunstancial e situacional e pressupõe um campo de incertezas e conflitos permanentes (ALVES; SOUZA, 1999, p. 133).

$\mathrm{O}$ estudo sobre os itinerários terapêuticos tem sua origem localizada na concepção, criada por Mechanic e Volkart (1960), de comportamento do enfermo, ou illness behaviour. Essa teoria pressupõe que a escolha por um tratamento é realizada racionalmente pelo individuo, com o objetivo de satisfazer suas necessidades. Para Alves e Souza (1999), trata-se de uma "concepção voluntarista, racionalista e individualista, com base no pressuposto de que as pessoas avaliam suas escolhas em termos de custobenefício" (p. 126).

Já uma segunda vertente dos estudos sobre comportamento do enfermo buscou investigar a influência exercida pelos valores culturais na escolha de respostas para os problemas de saúde, identificando os fatores culturais e/ ou elementos cognitivos que determinavam a busca por determinado tipo de tratamento ou agência de saúde. 
A maior contribuição dos estudos sobre o comportamento do enfermo foi a consideração dos fatores extrabiológicos da doença. Entretanto, segundo Alves e Souza (1999), a maior parte desses estudos apresentou graves problemas teórico-metodológicos, como a não diferenciação entre indivíduos que estavam em tratamento e aqueles que já haviam finalizado seus processos terapêuticos, o que não possibilitou avaliar as diferenças epistemológicas e implicações para a análise em ambos os casos. Além disso, esses estudos consideraram o modelo biomédico como único existente para compreensão das definições populares sobre doença e tratamento, não levando em consideração o conhecimento leigo e não racional utilizado pelo individuo no momento de escolha do tratamento.

Foi só a partir da década de 1970 que os trabalhos etnográficos passaram a salientar o fato de que

... as sociedades, e mesmo grupos sociais dentro delas, não só elaboram diferentes concepções médicas sobre causas, sintomas, diagnósticos e tratamentos de doenças, como estabelecem convenções sobre a maneira como os indivíduos devem comportar-se quando estão doentes (ALVES; SOUZA, 1999, p.127).

A partir desses estudos, foi possível reconfigurar a análise dos itinerários terapêuticos, que passou a incorporar os aspectos cognitivos e interativos envolvidos no processo de escolha do tratamento.

Diversos estudiosos sugeriram sistemas de classificações das diferentes interpretações sobre doenças e processos de tratamento. O esquema proposto por Kleinman (1978) é o mais utilizado e aceito atualmente. O autor propõe que os cuidados em saúde podem ser localizados em três subsistemas diferentes, dentro dos quais a experiência da doença é vivenciada: profissional, folk e popular. O subsistema profissional é composto pela medicina cientifica e pelos sistemas médicos tradicionais (chinês, ayurvédico etc); O folk é composto por curandeiros, rezadores, benzedeiros e outros; e o popular diz respeito ao campo leigo, no qual estão localizados a automedicação, a ajuda de amigos e familiares, a assistência mútua etc. Para a compreensão dessas práticas, Kleinman desenvolveu o conceito de "modelo explicativo", que pode ser definido como "um conjunto articulado de explicações sobre doença e tratamento, que determina o que se pode considerar como evidência clínica relevante e como se organiza e interpreta esta evidência com base em racionalizações construidas por perspectivas terapêuticas distintas" (ALVES; SOUZA, 1999, p.129).
Conforme discutido acima, o conceito de itinerários terapêuticos e seu uso tanto na pesquisa quanto na prática clínica é recente e ainda encontra diversas questões de cunho teórico-metodológico. Neste sentido, é necessário aprofundar o conhecimento sobre como as pessoas constroem suas trajetórias em busca de tratamento para suas aflições e, mais que isso, é necessário compreender quais são os elementos que influenciam suas escolhas e que adquirem importância no percurso do tratamento. A partir dessa compreensão, os itinerários terapêuticos podem ser considerados recursos importantes para compor a construção de projetos terapêuticos cuidadores que considerem como elementos o conjunto de recursos, experiências e significados de cada pessoa e que tenham como foco central seu contexto real de vida.

\section{CONSIDERAÇÕES FINAIS}

Embora ainda não constituindo uma cultura hegemônica, a busca da transformação dos modos de cuidar em saúde se constitui em um forte movimento para o qual convergem esforços de segmentos identificados com esse ideário. São exemplos desse esforço: as mudanças curriculares propostas para todos os profissionais da saúde, os programas de cuidado comprometidos com o desenvolvimento de uma abordagem integral, as discussões em torno às concepções inovadoras sobre o trabalho em equipe, $o$ esforço conjunto das associações de ensino das profissões da saúde na sensibilização e aprofundamento sobre as formas do cuidado integral e do trabalho em equipe, a constituição das redes de serviços de saúde mental, as novas abordagens necessárias ao desenvolvimento da estratégia de saúde da família, dentre outros. Nesses processos se identifica claramente a promoção de rupturas e inovações na prática da saúde.

Já existem muitos consensos teóricos, mas ainda há imensos obstáculos a serem superados presentes no cotidiano das práticas de cuidado e formação profissional, dentre eles o mais grave talvez seja a heterogeneidade da formação técnica e a multiplicidade de linguagens tradicionais em disputa no agir em saúde e a conseqüente carência de uma linguagem capaz de unificar algumas estratégias de abordagem ainda presente em muitos cenários assistenciais.

Nessa direção se espera que os apontamentos aqui apresentados possam contribuir na discussão e na prática das equipes e profissionais que buscam caminhos para os impasses atuais do campo da saúde. 
MÂNGIA, E. F.; MURAMOTO, M. T. Therapeutic itinerary and the construction of caring therapeutic projects. Rev. Ter. Ocup. Univ. São Paulo, v. 19, n. 3, p. 176-182, set./dez. 2008.

\begin{abstract}
S: The construction of new caring approaches in health field demands our attention in a context of changes and challenges evocated by the consolidation, maintenance and improvement of SUS. In this perspective, it is necessary the incorporation of new resources, capable of contribute to the construction and elaboration of intervention proposals centered in individuals and that consider their interaction with context and lives history. By using the model proposed by Merhy, in which the author defends that the construction of new approaches must be based on relational universe, where it is possible to produce the "caring that cares", based particularly on soft technologies, we introduce the concept of therapeutic itineraries and discuss the comprehension of this phenomenon as a resource that provides the construction of caring therapeutic projects regarding the person's experiences, meanings, paths, wishes and necessities on the elaboration of his own treatment process.
\end{abstract}

KEY WORDS: Delivery of health care. Health services accessibility. Program evaluation.

\title{
REFERÊNCIAS
}

ALVES, P. C. B.; SOUZA, I. M. A. Escolha e avaliação de tratamento para problemas de saúde: considerações sobre o itinerário terapêutico. In: RABELO, M. C. M.; ALVES, P. C. B.; SOUZA, I. M. A. Experiência de doença e narrativa. Rio de Janeiro: Fiocruz, 1999.

FRANCO, T. B. Processos de trabalho e transição tecnológica na saúde: um olhar a partir do Sistema Cartão Nacional de Saúde. In: Pensar BH: política social, n.8. Belo Horizonte, 2003.

MÂNGIA, E. F. Contribuições da abordagem canadense "Prática de Terapia Ocupacional Centrada no Cliente" e dos autores da desinstitucionalização italiana para a Terapia Ocupacional em Saúde Mental. Rev. Ter. Ocup. Univ. São Paulo, v. 13, n. 3, p. 127-134, 2002.

MÂNGIA, E. F.; MURAMOTO, M. T.: O estudo de redes sociais: apontamentos teóricos e contribuições para o campo da saúde. Rev. Ter. Ocup. Univ. São Paulo, v. 16, n. 1, p. 22-30, 2005.

MÂNGIA, E. F.; CASTILHO, L. P. V.; DUARTE, V. R. E. A construção de projetos terapêuticos. Rev. Ter. Ocup. Univ. São Paulo, v. 17, n. 2, 2006.

MÂNGIA, E. F.; MURAMOTO, M. Integralidade e construção de novas profissionalidades nos contexto dos serviços substitutivos de saúde mental. Rev. Ter. Ocup. Univ. São Paulo, v. 17, n. 3, p. 115-122, 2006.

MÂNGIA, E F.; MURAMOTO, M. Redes sociais e construção de projetos terapêuticos: um estudo em serviço substitutivo em saúde mental. Rev. Ter. Ocup. Univ. São Paulo, v. 18, n. 2, p.
54-62, 2007.

MÂNGIA, E. F.; YASUTAKI, P. M.Itinerários terapêuticos e novos serviços de saúde mental. Rev. Ter. Ocup. Univ. São Paulo, v. 19, n. $1,2008$.

MÂNGIA, E. F.; MURAMOTO, M. T.; SELMA, L. Classificação Internacional de Funcionalidade e Incapacidade e Saúde (CIF): processo de elaboração e debate sobre a questão da incapacidade. Rev. Ter. Ocup. Univ. São Paulo, v. 19, n. 2, p. 121-130, 2008.

MURAMOTO, M. T.; MÂNGIA, E. F. A sustentabilidade da vida cotidiana: um estudo das redes sociais de usuarios de servico de saúde mental no município de Santo André. Revista Ciência e Saúde Coletiva, Rio de Janeiro, 2009. Disponivel em: http:// www.abrasco.org.br/cienciaesaudecoletiva/artigos/artigo_int. php?id artigo $=3263$.

MERHY, E. E. Em busca do tempo perdido: a micropolítica do trabalho vivo em ato, em saúde. In. MERHY, E. E.; ONOCKO, R. (orgs.). Agir em saúde. Um desafio para o público. São Paulo: Hucitec, 1997.

MERHY, E. E.; FRANCO, T. B , Por uma composição técnica do trabalho centrada nas tecnologias leves e no campo relacional. In: Saúde em Debate, Rio de Janeiro, v. 27, n. 65, 2003.

MERHY, E. E. Engravidando palavras: o caso da integralidade. Palestra proferida no Congresso da Rede Unida, Belo Horizonte, 2005. Disponível em [http://www.uff.br/saudecoletiva/professores/ merhy/capitulos-06.pdf]. 